\title{
Preparatory Activations across a Distributed Cortical Network Determine Production of Express Saccades in Humans
}

\author{
Jordan P. Hamm, ${ }^{1,2}$ Kara A. Dyckman, ${ }^{3}$ Lauren E. Ethridge, ${ }^{1,2}$ Jennifer E. McDowell, ${ }^{1,2}$ and Brett A. Clementz ${ }^{1,2}$ \\ Departments of ${ }^{1}$ Psychology and ${ }^{2}$ Neuroscience, BioImaging Research Center, University of Georgia, Athens, Georgia 30602, and ${ }^{3}$ Department of \\ Psychiatry, Massachusetts General Hospital, Boston, Massachusetts 02129
}

\begin{abstract}
Reaction time variability across trials to identical stimuli may arise from both ongoing and transient neural processes occurring before trial onset. These processes were examined with dense-array EEG as humans completed saccades in a "gap" paradigm known to elicit bimodal variability in response times, including separate populations of "express" and regular reaction time saccades. Results indicated that express reaction time trials could be differentiated from regular reaction time trials by (1) pretrial phase synchrony of occipital cortex oscillations in the $8-9 \mathrm{~Hz}$ (low alpha) frequency range (lower phase synchrony preceding express trials), (2) subsequent mid- and late-gap period cortical activities across a distributed occipital-parietal network (stronger activations preceding express trials), and (3) posttarget parietal activations locked to response generation (weaker preceding express trials). A post hoc path analysis suggested that the observed cortical activations leading to express saccades are best understood as an interdependent chain of events that affect express saccade production. These results highlight the importance of a distributed posterior cortical network, particularly in right hemisphere, that prepares the saccade system for rapid responding.
\end{abstract}

\section{Introduction}

Multimodal reaction time distributions to identical stimuli may indicate important features of neural processing dynamics. A classic example of such phenomena is the "gap effect" in the saccade literature (Saslow, 1967; Fischer and Boch, 1983; Dorris and Munoz, 1995). Visually guided saccades normally occur between 170 and $230 \mathrm{~ms}$ (Fischer et al., 1993) and are initiated by signals from frontal and parietal ocular-motor cortices to brainstem nuclei (Leigh and Zee, 2006). One means of eliciting faster responses is to separate the fixation point and saccadic target by a subsecond blank screen, or "gap." Under such conditions, primates, including humans, have a bimodal latency distribution, including a population of "express saccades" with a mode occurring 90-120 ms after target appearance, and a second population of more regular reaction time saccades (Fischer et al., 1993; Clementz, 1996).

Investigations of the gap effect have revealed important characteristics of superior colliculus (SC) functioning that support rapid responding. For instance, the pattern of tonic activity across the SC ocular-motor map determines saccadic reaction time (Dorris et al., 1997). If SC buildup cell activity is augmented before onset of a visual target (e.g., insertion of a gap), a visually guided saccade can be generated early; otherwise, saccadic re-

Received Feb. 12, 2010; revised March 29, 2010; accepted April 3, 2010.

This work was supported by National Institutes of Health Grants MH076998 and MH082514. We thank Noah Duncan and Emily Leonard Parks for their help with data collection.

Correspondence should be addressed to Brett A. Clementz, Psychology Department, Psychology Building, Baldwin Street, University of Georgia, Athens, GA 30602. E-mail: clementz@uga.edu.

D0I:10.1523/JNEUROSCI.0785-10.2010

Copyright $\odot 2010$ the authors $\quad 0270-6474 / 10 / 307350-08 \$ 15.00 / 0$ sponses are associated with motor-cortical signaling and are of normal latency (Edelman and Keller, 1996; Munoz and Fecteau, 2002).

Investigations of cortical mechanisms underlying gapinduced bimodality in latency distributions have primarily involved single-unit recordings of frontal eye fields in monkeys (Dias and Bruce, 1994; Everling and Munoz, 2000) and electroencephalography (EEG) measurements without respect to cortical sources of signals measured at the sensors (Everling et al., 1996). The neurophysiology studies have identified gap period firing in cortical neurons specific to express saccades, and EEG research confirmed in humans the existence of unspecified gap period preparatory processes peculiar to express saccades. It is likely that many cortical locations simultaneously contribute to the gap phenomenon, consistent with there being multiple cortical inputs to brainstem ocular-motor nuclei (Leigh and Zee, 2006). Understanding the distributed nature of presaccadic activities would provide important information regarding how the cerebral cortex, in a few hundred milliseconds, can register a transient cue, prepare sensory processing centers, and prime subcortical nuclei to enable rapid action.

An additional focus of the current study concerns the stochastic nature of saccadic gap effects. Latencies in the gap task depend on experience and context (Paré and Munoz, 1996), but it remains unclear why, even after considerable practice, participants generate express saccades on only a proportion of trials (Fischer and Ramsperger, 1986). Power and/or phase in the alpha band before trial onset may influence visual target detection (Ergenoglu et al., 2004; Busch et al., 2009; Mathewson et al., 2009), and the nature of such occipital cortex activity in the pregap period 


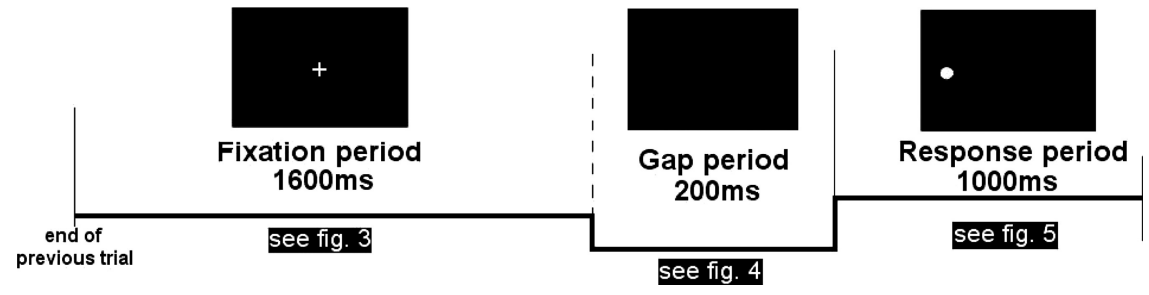

Figure 1. Stimuli and analysis periods. Results from each time period are presented in the figures referenced at that segment.

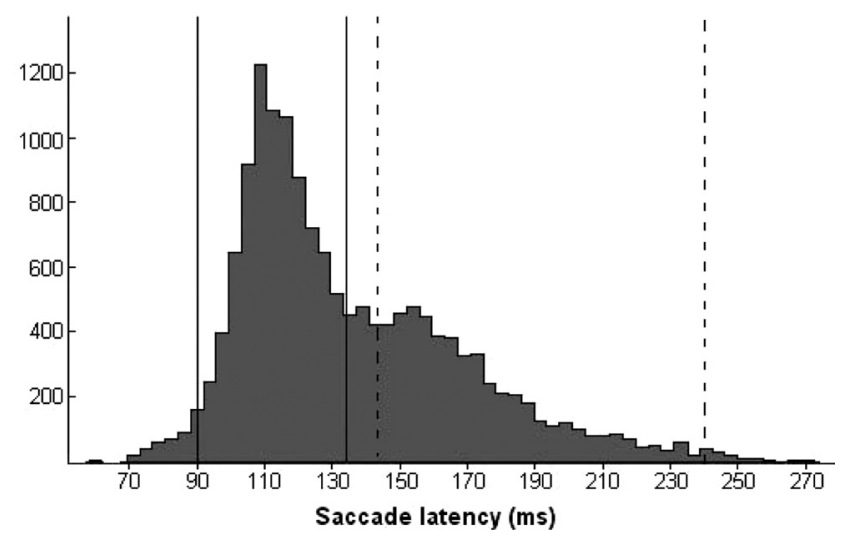

Figure 2. Reaction time histogram of all saccades made by all participants. The solid lines indicate express saccade range $(90-135 \mathrm{~ms})$, and the dotted lines indicate regular saccade range (145-240 ms).

could help determine saccadic latency variability. The influence of such a mechanism on saccadic latency variability in the gap paradigm has yet to be investigated. The present study used dense-array EEG and analysis of data in source space to investigate these phenomena.

\section{Materials and Methods}

\section{Participants}

Female participants $(N=15)$ were recruited through the undergraduate psychology research pool at the University of Georgia (mean age, 19.5 years; SD, 1.2). Subjects were in good physical health, absent of known neurological hard signs, and provided informed consent. Exclusion criteria, which were assessed via individual interviews, included neurological disease, a history of severe head trauma or brain injury, and current or past drug or alcohol abuse. This project was approved by the University of Georgia Institutional Review Board.

\section{Stimuli and procedure}

Stimuli (Fig. 1) were presented on a 21 inch flat-surface high-resolution color monitor ( $60 \mathrm{~Hz}$ vertical refresh) situated $100 \mathrm{~cm}$ from the participant's nasion. The task and visual stimuli were the same for all trials. To start the trial, a fixation cross within a square $\left(2.5^{\circ}\right)$ was presented at the center of the monitor and remained for $1600 \mathrm{~ms}$. The center stimulus was extinguished and the screen remained blank for $200 \mathrm{~ms}$ (gap), at which point a $2.5^{\circ}$ gray ring was presented for $1000 \mathrm{~ms}$ in one of two locations $\left( \pm 8^{\circ}\right.$ from fixation; randomly determined) in the horizontal plane (onehalf in each visual field). Participants were instructed to move their eyes to the ring as quickly and accurately as possible. Participants completed 144 saccade trials per session over four sessions.

\section{Electrophysiological recording and data preprocessing}

Data collection. EEG data were vertex-referenced and recorded using a 256-sensor Geodesic Sensor Net and NetAmps 200 amplifiers (EGI). Individual sensor impedances were kept below $50 \mathrm{k} \Omega$ (Ferree et al., 2001). In addition, an electrolyte bridge test was conducted between all pairs of sensors before recording (Tenke and Kayser, 2001), and if there was evidence of bridging, sensors were adjusted until bridging was no longer evident (this was rarely required). Data were sampled at $500 \mathrm{~Hz}$ with an analog filter bandpass of 0.1-200 Hz. Sensors located at the outer canthi of each eye and below and above both eyes recorded horizontal and vertical eye movements and eye blinks. After data collection, the three-dimensional locations of sensors were acquired using a photogrammetry rig (EGI).

Eye movement data. The position data from the horizontal eye sensors for individual trials were visually inspected and scored for saccadic latencies (time from peripheral target appearance to saccade initiation) for each trial as by Dyckman and McDowell (2005) using routines written in MATLAB (The MathWorks). Trials with blinks in the gap and late pregap period (from $350 \mathrm{~ms}$ before target appearance until saccade onset), and trials with no saccades, were eliminated (17 per session on average). Additionally, eye movements in the pregap fixation period (500 $\mathrm{ms}$ after fixation appearance to fixation disappearance) were quantified and compared between saccade types. Express and regular saccades did not differ in the percentage of trials containing blinks $\left(t_{(14)}=1.05 ; p=0.310\right.$; two-tailed $)$, and there was no evidence of horizontal or vertical saccades in this time range. A reaction time histogram was created to determine outlier values and to confirm modes for express and regular saccades in the distributions (see Fig. 2).

EEG data. EEG sensors located at the neck and cheeks were excluded, leaving 211 sensors for data analyses. Raw data were visually inspected offline for bad sensor recordings (BESA 5.1; MEGIS Software). Bad sensors (no more than $5 \%$ of sensors for any subject) were interpolated using spherical spline interpolation method of BESA. Cardiac artifacts were eliminated using the Independent Component Analysis module in EEGLAB 6.0 (Delorme and Makeig, 2004). The artifact-free data were then transformed to an average reference.

\section{Data analysis}

Bimodality in saccadic latency distributions. For confirmation of the production of express saccades in all participants, the following steps were taken (for an example, see also Clementz, 1996). For each participant, reaction times within the defined range of 90-240 ms were standardized (493 trials on average), adjusted for skewness using the generalized BoxCox power transformation (Box and Cox, 1964), and tested for bimodality using the SKUMIX algorithm (MacLean et al., 1976). For tests of bimodality (admixture) within response latency distributions for each participant, $F$ values for three- (unimodal distribution) and fiveparameter (bimodal distribution) models generated by SKUMIX were compared using a $\chi^{2}$ test on $1 \mathrm{df}$. Associated values of $p<0.05$ indicated the presence of two modes. After these analyses, individual model parameters (means and SDs for the two modes) were averaged to produce a model of the latency distributions that informed the definitions of express and regular saccades used for subsequent analyses.

Pregap period. To investigate the possibility that neural activity before trial onset may influence gap onset detection and subsequent saccadic reaction time, the following procedure was used. Single-trial voltage data from $950 \mathrm{~ms}$ before to $50 \mathrm{~ms}$ after gap onset ( 500 samples) was multiplied by a 500 point Hanning window (this period was extended $50 \mathrm{~ms}$ into gap period to account for attenuated activity at the edge as a consequence of windowing). Single-trial data for each sensor were then Fourier-transformed to $1 \mathrm{~Hz}$ resolution using the fast Fourier transform (FFT) algorithm in MATLAB. Single-trial power was obtained by squaring the absolute value of the complex result of the FFT. Intertrial coherence (ITC) (a measure of similarity in phase of oscillatory activity over trials in relation to gap onset) (Busch et al., 2009) was calculated by dividing the complex result of the FFT by its absolute value (the amplitude) for each trial and sensor. These values were then summed across trials within subjects and divided by the corresponding number of trials. Resulting values were the Rayleigh statistic $R$, which is bound between 0 and 1 ( 1 indicating perfect phase alignment across trials). Pregap power and ITC of individual sensors were compared between express and regular saccades within subjects.

To explore differences in ITC found between express and regular saccades, trials were segregated into four phase bins (0-90, 90-180, $180-$ 
A
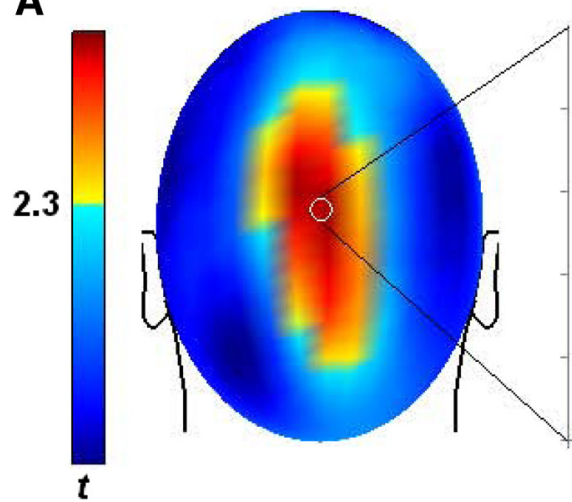

$t$

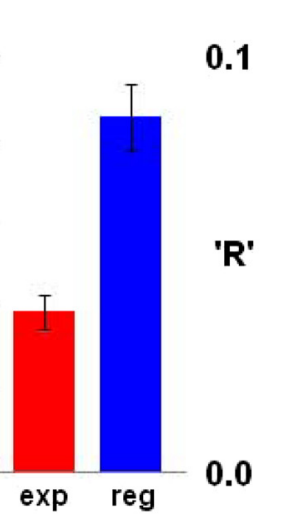

B

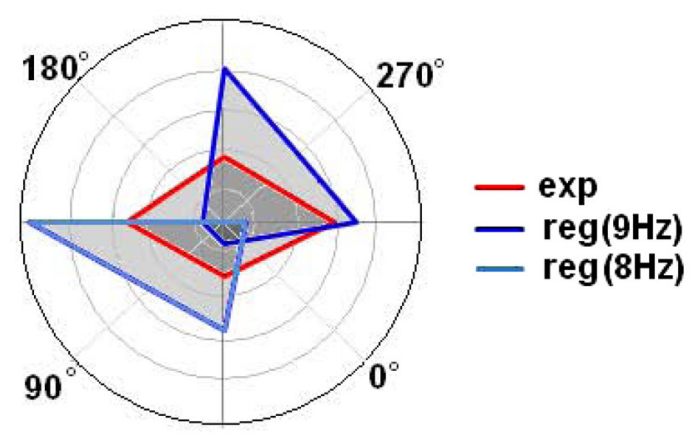

15

10

5

1

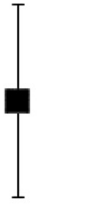

mean
$\%$ express
-5

$-10$

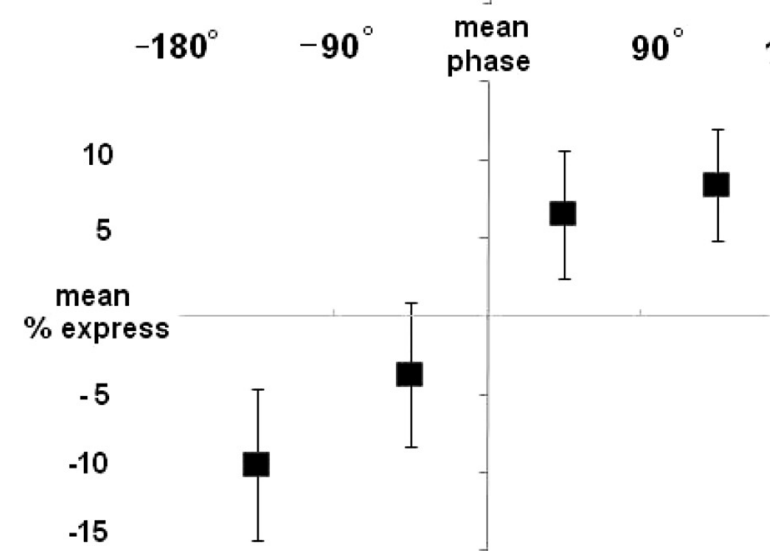

Figure 3. Pregap period: two-dimensional topographies reveal significant $t$ values in warm colors; $8-9 \mathrm{~Hz}$ intertrial phase coherence (with respect to fixation disappearance) is higher for regular saccades (graph represents data from $0 \mathrm{z}$ ) $(\boldsymbol{A}) ; 8-9 \mathrm{~Hz}$ phase is random across express, but not regular saccade trials $(\boldsymbol{B})$; trials with phase values below mean phase at $8 \mathrm{~Hz}$ have higher proportions of express saccades, and trials with phase values above mean phase at $9 \mathrm{~Hz}$ have higher proportions of express saccades (C). Error bars reflect within-subject SE (Loftus and Masson, 1994).

$270,270-360^{\circ}$; mean phase for a subject, $0^{\circ}$ ) for 8 and $9 \mathrm{~Hz}$ activity recorded at $\mathrm{Oz}$ (central sensor in significant ITC cluster) in this pretrial period. Percentage of saccades in the express range were calculated for each phase bin for each participant, and these data (deviation scores from mean percentage express saccades) were subject to a 4 (phase bin) $\times 2$ (express, regular) two-way repeated-measures ANOVA.

Gap period analyses. To investigate neural activities during the $200 \mathrm{~ms}$ gap period, EEG data from $150 \mathrm{~ms}$ before gap onset to $50 \mathrm{~ms}$ after target appearance ( $400 \mathrm{~ms}$ total) were baseline adjusted using the $150 \mathrm{~ms}$ pregap period and averaged over trials using BESA 5.1. Both subsequent target directions were averaged together since, before target occurrence at the end of the gap period, subjects did not know direction of the required response. Voltage data were collapsed into bins of $10 \mathrm{~ms}$ duration before making saccade-type comparisons.

Response period analyses. To investigate neural activities specifically related to saccade generation, response-locked data (from $250 \mathrm{~ms}$ before to $100 \mathrm{~ms}$ after saccade initiation) were baseline-adjusted using the 150 $\mathrm{ms}$ pregap period and were averaged over trials using BESA 5.1. As can be seen from Figure $5 A$, the saccade voltage spike started $15 \mathrm{~ms}$ before the peak saccadic response. As a result, voltage data were analyzed using the same procedure as described for the gap period analyses only up to $15 \mathrm{~ms}$ before the saccade voltage peak in the response-locked averages.

Statistical criteria. Paired-samples $t$ tests $(n=15$; two-tailed) were conducted separately for each sensor for comparing power, ITC, gap period voltages, and response period voltages between saccade types. For significance thresholding, a clustering method (Forman et al., 1995) was used to take account of the nonindependence of data from adjacent EEG sensors with significance levels determined based on the noise level of the data (estimated from the prestimulus period) (for examples, see Clementz et al., 2008; Krusemark et al., 2008) and Monte Carlo simulations calculated using AlphaSim (Cox, 1996). To maintain the family-wise $\alpha<$ 0.01 , individual $t$ tests for a given sensor required at least six neighboring sensors with effects statistically significant at $p<0.035$.
Source analyses. After analyses calculated on voltage data at the sensors, standardized low-resolution brain electromagnetic tomography (sLORETA) (Pascual-Marqui, 2002) was used to estimate brain regions involved in determining the between-saccade-type differences on EEG signals observed in the sensor space data (Clementz et al., 2007; Krusemark et al., 2008). sLORETA is a modification of minimum norm least squares (Hämäläinen and Ilmoniemi, 1994) that uses the standardization of the minimum norm inverse solution to infer highprobability regions of brain activation given the measured EEG data. sLORETA solutions yield pseudostatistics that are not appropriate for determining strength of activity, but they provide accurate information about the regions of activity that can account for the voltage pattern recorded at the sensors (Soufflet and Boeijinga, 2005).

An averaged magnetic resonance (MR) image from the Montreal Neurological Institute (Collins et al., 1994) was used to construct a threecompartment boundary element method (BEM) model (Fuchs et al., 2001) before source localization. The MR images were segmented into skin surface, inside of the skull, and cortex. Standard homogeneous conductivities were assumed for the skin, skull, and brain $(0.33,0.0042$, $\left.0.33^{-1} \cdot \mathrm{m}^{-1}\right)$. For this BEM model, the average triangle edge lengths were $9 \mathrm{~mm}$ for the skin, $7.1 \mathrm{~mm}$ for the skull, and $5 \mathrm{~mm}$ for the brain compartment. Sensors were mapped to the head surface by first matching the fiducial locations (nasion, left and right preauricular points) from the EEG data collection session to the fiducial locations on the averaged segmented skin surface and then projecting the sensors to the skin surface using a nonlinear least-square fitting procedure. The sLORETA calculations then were obtained for each saccade type for each individual. Differences between saccade types were calculated for each individual and averaged to produce source difference maps in brain space. Response period difference maps were created for left and right targets separately. These source localization procedures were implemented using CURRY (version 5.0; Neuroscan). To determine whether these source estimations represented unique or equivalent cortical generators for the EEG 


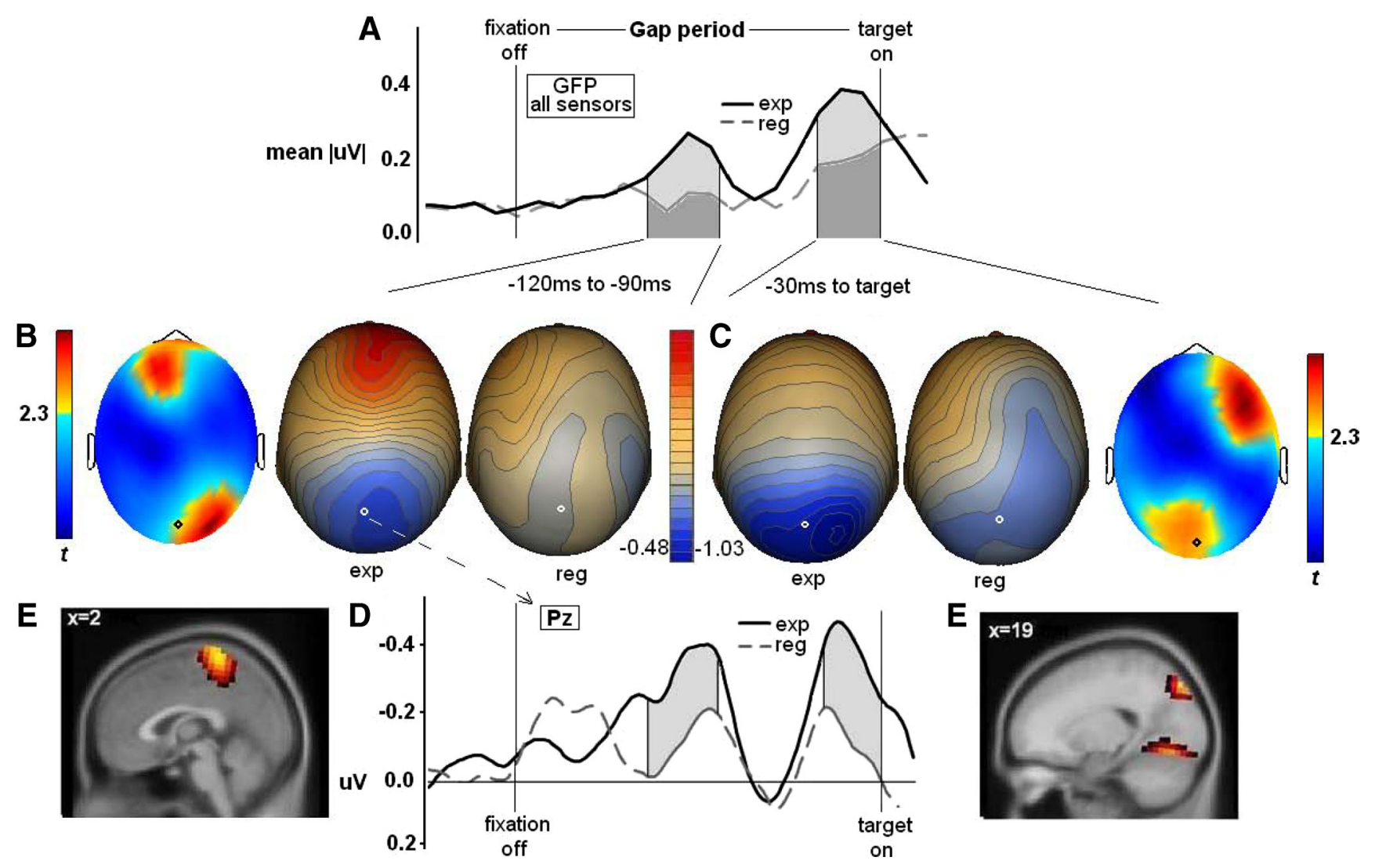

Figure 4. Gap period analyses: time by amplitude plots show global field power over all sensors, indicating two significant voltage peaks (A); two-dimensional topographies reveal significant $t$ values in warm colors for mid-gap $(\boldsymbol{B})$ and late-gap ( $\boldsymbol{C}$ periods, with corresponding voltage topographies (B, $(\mathbf{C}$; event-related potential data from Pz sensor (indicated on heads) (D); expressregular source difference maps ( $F$-distributed) on the MNI brain thresholded at $>2$ SDs above the mean voxel strength for each time period $(\boldsymbol{E})$.

voltage differences recorded at separate time points during a trial, local maxima of each sLORETA solution (mid-gap, late-gap, left target response period, and right target response period) in parietal cortex were identified for each participant and compared using separate one-way repeated-measures ANOVAs for $x-z$ coordinates.

Post hoc path analyses. For each participant, ITC, mid-gap voltage, and late-gap voltage values were averaged across their respective significant clusters to produce single values for a path analysis along with percentage express saccade production (LISREL; Scientific Software International). All temporally plausible path models were analyzed and compared on $\chi^{2}$, and parsimonious goodness-of-fit index (Mulaik et al., 1989) to identify the model that best explained the intervariable relationships.

\section{Results}

\section{Behavior}

All participants produced saccades with a latency mode in the express range of 90-120 ms as defined in the literature (Fischer and Boch, 1983; Fischer et al., 1993; Clementz, 1996), and most participants (13 of 15) produced response latencies with significantly bimodal distributions. The two subjects without significantly bimodal distributions produced primarily express saccades. A response latency histogram for all saccades for all participants (Fig. 2) confirmed by visual inspection the existence of separate peaks for express and regular saccades. The estimated mean express saccade mode across subjects was $112.8 \mathrm{~ms}$ (SD, 12.2), and the estimated mean regular reaction time saccade mode was 164.2 ms (SD, 22.7). Based on the estimated SKUMIX latency mode parameters and the histogram of saccadic response latencies (Fig. 2), and in concordance with previous studies (Fischer et al., 1993; Clementz, 1996), saccade types were defined for all participants:
(1) express saccades had latencies between 90 and $135 \mathrm{~ms}$, and (2) regular saccades had latencies between 145 and $240 \mathrm{~ms}$. The following analyses of brain activity are based on this classification.

\section{Brain activity}

Pregap period

For express and regular saccade trials, alpha-band $(8-12 \mathrm{~Hz})$ power (in square microvolts) was identically distributed across the back of the scalp for occipital and parietal sensors (Fig. 3A) during the pregap period; paired-samples $t$ tests $(\mathrm{df}=14$, twotailed) indicated no significant difference in power between saccade types for any alpha-band frequency $(p>0.035$ for all sensors). The two saccade types, however, did differ on ITC for both 8 and $9 \mathrm{~Hz}$ during this time range ( $\mathrm{df}=14$, two-tailed) (Fig. $3 A$ ). Before gap period onset, on trials that would subsequently yield a regular reaction time saccade, participants had significantly larger ITC values than on trials that would subsequently yield an express saccade (averaged across significant sensors for both 8 and $9 \mathrm{~Hz}$ ITC). Radial plots of pregap 8 and $9 \mathrm{~Hz}$ phase from the significant sensor cluster over posterior cortex (Fig. 3B) indicate that pregap phase was essentially random over trials on which subjects eventually produced an express saccade, but had specific phase characteristics as a function of frequency for trials on which subjects eventually produced a regular reaction time saccade. Subsequent two-way repeated-measures ANOVA results revealed a phase by frequency interaction $\left(F_{(3,42)}=2.88 ; p=\right.$ $0.047)$, indicating that proportion of express saccades differed between phase bins across frequencies (Fig. 3C). 


\section{Gap period}

Paired-samples $t$ tests ( $\mathrm{df}=14$, twotailed) were used to compare neural activities between trials that would subsequently yield express versus regular saccades. There were two time ranges that differentiated groups (Fig. $4 A$ ); the voltage topographies within saccade type remained constant within each time range. The first difference occurred from 80 to $110 \mathrm{~ms}$ into the gap (mid-gap period) across middle and right parietal and occipital sensors and surrounding $\mathrm{FCz}$ (Fig. $4 B)$; the second difference occurred from 170 to $200 \mathrm{~ms}$ into the gap (at the very end of the gap; late-gap period) across middle parietal, occipital, and right frontal sensors (Fig. 4C). No other time bins yielded significant between-saccade type differences. For both time periods, trials producing express saccades had greater evoked voltage difference from baseline than trials producing regular reaction time saccades, which is reflected in two positive peaks in sensor $\mathrm{Pz}$ (Fig. 4D).

Source analyses indicated that neural activations in specific brain regions differentiated between saccade types (Fig. $4 E$ ). For the mid-gap period, trials on which there would be express saccades had higher activity in superior dorsal medial parietal lobe (Brodmann's area 7). In lategap period, neural activation in two distinct brain regions distinguished trials leading to express versus regular saccades: (1) the right posterior cortex at the junction of parietal and occipital cortices (at the junction of Brodmann's areas 7 and 19) and (2) bilateral calcarine fissure in primary visual cortex (Brodmann's area 17). In both instances, express saccade trials had greater cortical activity than regular saccade trials.

\section{Response period}

Paired-samples $t$ tests $(\mathrm{df}=14$; two-tailed) (Fig. $5 B)$ were used to compare neural activities between express and regular saccade trials for the period preceding saccade onset. Mid-central, parietal, occipital, and right temporal and right frontal sensor voltages during the time period immediately before saccade initiation (from 45 to $15 \mathrm{~ms}$ before onset of the response) (Fig. $5 A, B$ ) significantly differentiated saccade types for both leftward and rightward responses. The voltage topographies within saccade type remained constant within this time range and had marginally different distributions for left and right targets (topography combined for left and right targets for simplicity of presentation) (Fig. 5B). No other time ranges yielded significant between-saccade type differences. Cortical activity immediately preceding regular reaction time saccades was stronger than immediately preceding express saccades, which is reflected in a negative peak in $\mathrm{CPz}$ (Fig. $5 \mathrm{C}$ ). Source analyses indicated that neural activations in Brodmann's area 7 (regular-express differences plotted in Fig. 5D) differentiated saccade types as a function of saccade direction. In response to left targets, regular saccades

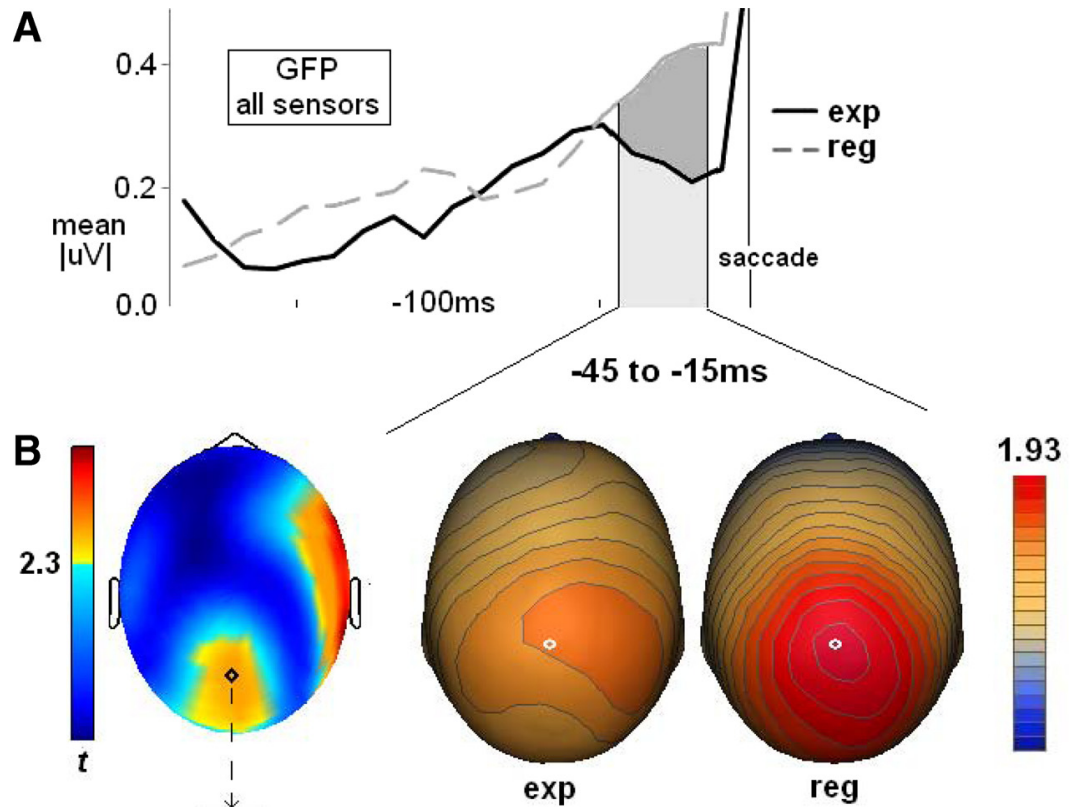

Figure 5. Response period: time by amplitude plots show global field power over all sensors, indicating a significant voltage peak before regular saccades (beginning of saccade spike is at far right) $(\boldsymbol{A})$; two-dimensional topographies reveal significant $t$ values in warm colors for this time period; corresponding voltage topographies (combined for left and right stimuli) (B); eventrelated potential data from (Pz sensor (indicated on heads) (C); express-regular source difference maps on the MNI brain thresholded at $>2$ SDs above the mean voxel strength for each stimulus location (D).

showed increased activity in right parietal cortex, and for right targets, regular saccades showed increased activity in left parietal cortex.

\section{Source location comparisons}

Repeated-measures ANOVAs for the parietal cortical locations of sLORETA solutions indicate that mid-gap, late-gap, left target response period, and right target response period signals originate from spatially distinct locations in terms of lateral $(x$ coordinate, $\left.F_{(3,42)}=3.29, p=0.030\right)$ and anterior-posterior distributions ( $y$-coordinate, $\left.F_{(3,42)}=3.56, p=022\right)$, but not dorsal-ventral distribution $\left(z\right.$-coordinate, $F_{(3,42)}=2.49, p=$ 0.073 ). Simple comparisons (paired-samples $t$ tests, $\mathrm{df}=14$, twotailed, 12 comparisons) revealed that laterality differences stem from the right target response period source in left hemisphere differing from all other activations. The anterior-posterior differences stem from the late-gap source being distinct from all other sources (the late-gap source is more caudal). The midgap and left-target response locked sources did not differ significantly in either laterality or anterior-posterior location (supplemental Fig. 1, available at www.jneurosci.org as supplemental material). 


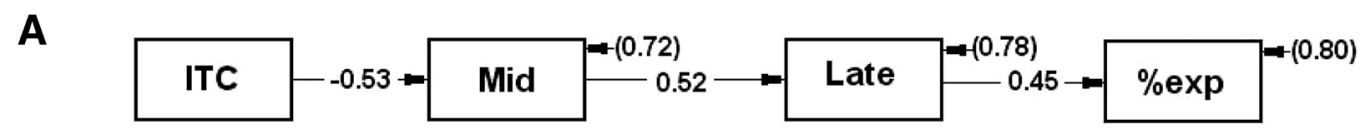

B

\begin{tabular}{|c|c|c|c|c|}
\hline Degrees of freedom & Model & Parsimonious Goodness of Fit & Goodness of Fit & chisquare(p) \\
\hline \multirow[t]{3}{*}{3} & {$[I T C]->[E]->[L]->[\% \exp ]$} & 0.29 & 0.96 & $1.14(.76)$ \\
\hline & {$[\mathrm{ITC}]>[\% \exp ] ;[\mathrm{E}]>>[\mathrm{L}]>[\% \exp ]$} & 0.26 & 0.87 & $4.31(.23)$ \\
\hline & {$[\mathrm{ITC}]>>[\% \exp ] ;[\mathrm{E}]->[\% \exp ] ;[\mathrm{L}]>>[\% \exp ]$} & 0.23 & 0.78 & $8.05(.05)$ \\
\hline \multirow[t]{4}{*}{2} & {$[\mathrm{ITC}]>[\mathrm{E}]->[\mathrm{L}]->[\% \exp ] ;[\mathrm{ITC}]>>[\% \mathrm{exp}]$} & 0.20 & 0.98 & $0.46(.79)$ \\
\hline & {$[\mathrm{ITC}]>[\mathrm{E}]->[\mathrm{L}]->[\% \exp ] ;[\mathrm{ITC}]>>[\mathrm{L}]->[\% \exp ]$} & 0.19 & 0.97 & $0.75(.68)$ \\
\hline & {$[\mathrm{ITC}]>[\mathrm{E}]>[\mathrm{L}]>[\% \exp ] ;[\mathrm{E}]>[\% \exp ]$} & 0.19 & 0.96 & $1.07(.58)$ \\
\hline & {$[\mathrm{ITC}]>[\mathrm{L}]>>[\% \exp ] ;[\mathrm{E}]->[\% \exp ] ;[$ ITC $]>[\% \exp ]$} & 0.16 & 0.81 & $6.69(.03)$ \\
\hline \multirow[t]{3}{*}{$\overline{\mathbf{I}}$} & {$[\mathrm{ITC}]->[\mathrm{E}]->[\mathrm{L}]->[\% \exp ] ;[\mathrm{E}]>>[\% \exp ] ;[\mathrm{ITC}]->[\% \exp ]$} & 0.10 & 0.99 & $0.39(.52)$ \\
\hline & {$[$ ITC $]>[\mathrm{E}]->[\% \exp ] ;[\mathrm{ITC}]->[\mathrm{L}]->[\% \exp ] ;[\mathrm{ITC}]->[\% \exp ]$} & 0.09 & 0.88 & $3.78(.05)$ \\
\hline & {$[\mathrm{E}]>[\mathrm{L}]>>[\% \exp ] ;[\mathrm{ITC}]->[\mathrm{L}]>[\% \exp ] ;[\mathrm{E}]>[[\% \exp ] ;[\mathrm{ITC}]>[\% \exp ]$} & 0.09 & 0.88 & $3.98(.04)$ \\
\hline
\end{tabular}

Figure 6. Post hoc path analyses of participant scores for intertrial phase coherence across occipital sensors [ITC], early/mid-gap peak amplitude [E], late-gap period peak amplitude [L], and proportion of saccades in the express range [\%exp]. $\boldsymbol{A}$, The displayed path was the best fitting and most parsimonious model. Estimated correlation coefficients are embedded in paths, and disturbances are in parentheses. $\boldsymbol{B}$, Also displayed are results associated with the next best fitting and parsimonious candidate path models (truncated from 20); they are organized by degrees of freedom and parsimonious goodness-of-fit index. The arrows indicate paths.

\section{Post hoc path analyses}

Of all path models evaluated (i.e., those fitting a logical temporal sequence with $\mathrm{df}>0)$ (Fig. 6), the best-fitting and most parsimonious model $\left(\chi^{2}=1.14 ; p=0.760\right.$; parsimony goodness-of-fit index $=0.29$ ) (Mulaik et al., 1989) had the form \{pregap ITC $\} \rightarrow$ $\{$ mid-gap EEG $\} \rightarrow$ \{late-gap EEG $\} \rightarrow$ [\%express saccades]. The only other model that approached the same combination of good fit and high parsimony included an additional path \{pregap ITC $\rightarrow$ [\%express saccades]. These best-fitting models were also significantly better than a simple multiple regression model for predicting percentage express saccades; including response-locked EEG significantly worsened all model fits.

\section{Discussion}

Four aspects of cortical activity differentiated express from regular latency saccades. Neural activities associated with saccade generation were in parietal-occipital cortical regions, highlighting the importance of a posterior cortical network, particularly in right hemisphere (Luna et al., 1998; Corbetta and Shulman, 2002), for preparing the saccade system for rapid responding. This obviously does not diminish the importance of subcortical (Edelman and Keller, 1996; Dorris et al., 1997; Munoz and Fecteau, 2002) and frontal cortical (Dias and Bruce, 1994; Everling and Munoz, 2000) brain regions for saccade generation generally, and express saccades particularly. In what follows, the distinct cortical activations will be discussed separately and then integrated to illustrate a coherent model associated with saccadic gap effects.

\section{Pretrial preparatory activity}

Pretrial oscillatory phase at $8-9 \mathrm{~Hz}$ over occipital cortex was more aligned to fixation disappearance (trial onset) before regular than express saccades. This could indicate that (1) a particular alpha-band phase window interferes with gap onset detection and, thus, with initiation of gap period brain processes necessary for express saccade production, and/or (2) express saccades result when occipital activity is less organized with respect to a pretrial fixation point.

The former possibility is consistent with research indicating neural oscillations mark rhythmic changes in cortical excitability (Ergenoglu et al., 2004; Jin et al., 2006; Busch et al., 2009). The current finding is also consistent with reports (Mathewson et al.,
2009) indicating a potent effect of pretrial alpha-band phase, especially in the low-alpha range (Busch et al., 2009), on performance in visual tasks. That ITC was higher on regular saccade trials is consistent with the account by Mathewson et al. (2009) of alpha as "pulsed inhibition" (i.e., alpha oscillations in particular phase windows diminish probability of target detection). The latter possibility is also consistent with associations between visual attention and stimulus-evoked neuronal entrainment (Schroeder and Lakatos, 2009). In the present study, neuronal entrainment to fixation onset as measured by ITC may index attentional engagement with the fixation point. Visual fixation is known to directly inhibit saccadic initiation (Munoz and Fecteau, 2002), so increased neural investment in fixation could delay neural activations necessary for saccade generation (Dorris et al., 1997).

Primates generate more express saccades with repeated exposure to gap tasks (Fischer and Ramsperger, 1986), which theoretically results from optimization of response preparation (Dorris and Munoz, 1998) and/or target detection with practice (Fine and Jacobs, 2002). Subjects in the present study altered pretrial alpha-band phase characteristics over sessions, with pretrial alpha-band ITC decreasing (sessions 1-4: 0.104, 0.093, 0.091, 0.079 ) and percentage express saccades increasing (sessions 1-4: $54,59,60,65 \%)$. These cross-session changes represent significant linear trends: $F_{(1,28)}=19.719, p<0.001$; and $F_{(1,28)}=30.08$, $p<0.001$, respectively. This may indicate that a mechanism by which humans speed response times is through early preparation of sensory systems for detection of change. Additional study of practice-induced changes in alpha-band phase may be a promising avenue for investigating behavioral plasticity associated with performance enhancements.

Roles for alpha-band oscillations in express saccade production have been suspected (Kirschfeld et al., 1996; Skrandies and Anagnostou, 1999). The current study did not find differences between preexpress and preregular saccade trials in alpha-band power, so the results are not consistent with the prediction by Kirschfeld et al. (1996) that alpha power at fixation disappearance predicts express saccade probability. Skrandies and Anagnostou (1999) used an analog of alpha-band phase (normalized amplitude), limited their investigation to high-range alpha frequencies $(11.72 \mathrm{~Hz})$, did not include pretrial or early-gap periods 
in their analyses, and found express-regular saccade differences at only one frontal and one parietal sensor. Multiple investigations indicate that the most profound effects of ongoing alpha are at lower alpha frequencies (Busch et al., 2009), before trial onset (Ergenoglu et al., 2004), and over occipital cortex (Capotosto et al., 2009; Mathewson et al., 2009).

\section{Gap period cortical activities}

At $80-110 \mathrm{~ms}$ into the gap period (mid-gap), superior parietal cortex activity increased on trials preceding express but not regular saccades. This region is associated with visual spatial attention and detection of stimulus spatial change (Corbetta et al., 1993). This neural activity may signal detection of fixation disappearance, a relevant visual-spatial cue before initiation of saccade preparation during gap paradigms.

At 170-200 ms into the gap period (late-gap), there were two cortical activations that differentiated express and regular reaction time saccade trials: primary visual cortex and inferior parietal cortex near the junction of Brodmann's areas 7 and 19 (in the putative location of human parietal eye fields) ( $\mathrm{McD}$ owell et al., 2008). In both instances, neural activity was higher on trials eventually producing express saccades. A similar pattern has been observed during sustained attention tasks, in which activity levels in specific neural ensembles precede presentation of relevant stimuli (Chawla et al., 1999; Driver and Frith, 2000; Pessoa et al., 2003) and influence subsequent behavioral responses.

The mid- and late-gap activations may prime saccadegenerating circuitry in anticipation of target appearance. Visual cortex has access to the brainstem saccade generators through the superior colliculus (Collins et al., 2005). Likewise, neurons in parietal eye fields have access to SC (Lynch et al., 1985), have influence on eye movements through this SC input (Keating et al., 1983), and discharge similarly, but not identically, to SC neurons during interstimulus gap periods (Paré and Wurtz, 2001; Ben Hamed and Duhamel, 2002). Given this circuitry, and the time course of the observed neural activations, these posterior cortex regions may prime brainstem nuclei necessary for express saccade production. This priming could involve the preparatory excitatory modulation of saccadic buildup neurons in SC necessary for express saccade generation (Dorris et al., 1997).

\section{Response-locked activity}

From 45 to $15 \mathrm{~ms}$ before saccade onset, neural activity in the hemisphere contralateral to the response direction, in the vicinity of precuneus, preceded regular but not express saccades. This same region is consistently activated during a number of saccade tasks (Dyckman et al., 2007; McDowell et al., 2008). The exact contribution of this region to saccadic generation is unclear, but the time course of its activation suggests a role in saccade triggering. This cortical activity marked a later processing stage necessary for the production of regular but not express saccades (which occurred $50 \mathrm{~ms}$ earlier on average). This finding supports the hypothesis that preparation for express saccades occurred at an earlier stage, most likely through priming of subcortical nuclei (Edelman and Keller, 1996; Dorris et al., 1997; Munoz and Fecteau, 2002), secondary to the gap period activations in visual and parietal cortices observed here (Fischer et al., 1993).

\section{Cortical network model}

The model that best explained the interrelations of ITC, mid-gap (superior parietal cortex) activity, late-gap (parietal eye field and visual cortex) activity, and proportion of express saccades produced was also the most temporally linear. This analysis sug- gested that the cortical activations leading to express saccades can be understood as occupying a single interdependent chain of events rather than as separate mechanisms that affect express saccade production independently. That is, disengaged (or random) low-frequency alpha-band activity predicts the amount of parietal signaling midway through the gap, which predicts visual cortical and parietal eye field activations later during the gap, which determines express saccade probability. Although this model will require verification in future studies, it converges with theory in many respects. For instance, similar to recent findings by Capotosto et al. (2009), this model maps visual-spatial event detection to the interplay of ongoing and transient occipital and parietal activities.

Similar to Everling et al. (1996) and Everling and Munoz (2000), this model, and the results overall, support the notion that express saccade generation is related to trial-to-trial variations in both ongoing and preparatory activities in cortical regions with access to the brainstem saccade-generating apparatus. Unlike those reports, our findings imply that express saccaderelated preparatory activities are in mainly parietal, not frontal, cortices. This seeming incongruence could be associated with multiple factors, including the following: (1) our use of densearray EEG recording and subsequent source estimation analyses that allowed for inference of location in brain space from where activity recorded at the sensors emanated [the data of Everling et al. (1996) may be consistent with the present findings if the same analyses with a similar sensor montage had been conducted, which was not technically feasible at the time]; and (2) the use by Everling and Munoz (2000) of single-unit recordings in frontal eye field of nonhuman primates, a technology much more sensitive to detecting neural activity regardless of whether it was locked to stimulus occurrence and/or response generation. Everling and Munoz (2000) did not measure activity from posterior brain regions, so the relationship between the frontal cortical signals they measured and the posterior cortical signals observed here is at present uncertain (but see Ruff et al., 2006; Ekstrom et al., 2008).

Nevertheless, the noninvasive methods of the current study for recording neural activity, and analyses of brain signals in the time, space, and frequency domains, provided a demonstration of the posterior cortical generators associated with express saccadic production. Similar to other recent visual processing investigations, these data highlight the importance of ongoing alpha-band phase on behavior and the role of posterior cortical structures in response preparation. It is possible that frontal cortical signals modulate the occipital-parietal preparatory activity observed here by providing an "attentional template" (Desimone and Duncan, 1995), in this case, one that decreases neural investment in central fixation during the pregap preparatory phase. This could be an exciting and important avenue to pursue in subsequent translational research.

\section{References}

Ben Hamed S, Duhamel JR (2002) Ocular fixation and visual activity in the monkey lateral intraparietal area. Exp Brain Res 141:512-528.

Box GEP, Cox DR (1964) An analysis of transformations. J R Stat Soc Series B Stat Methodol 26:211-252.

Busch NA, Dubois J, VanRullen R (2009) The phase of ongoing EEG oscillations predicts visual perception. J Neurosci 29:7869-7876.

Capotosto P, Babiloni C, Romani GL, Corbetta M (2009) Frontoparietal cortex controls spatial attention through modulations of anticipatory alpha rhythms. J Neurosci 29:5863-5872.

Chawla D, Rees G, Friston KJ (1999) The physiological basis of attentional modulation in extrastriate visual areas. Nat Neurosci 2:671-676. 
Clementz BA (1996) The ability to produce express saccades as a function of gap interval among schizophrenia patients. Exp Brain Res 111:121-130.

Clementz BA, Brahmbhatt SB, McDowell JE, Brown R, Sweeney JA (2007) When does the brain inform the eyes whether and where to move? An EEG study in humans. Cereb Cortex 17:2634-2643.

Clementz BA, Wang J, Keil A (2008) Normal electrocortical facilitation but abnormal target identification during visual sustained attention in schizophrenia. J Neurosci 28:13411-13418.

Collins CE, Lyon DC, Kaas JH (2005) Distribution across cortical areas of neurons projecting to the superior colliculus in New World monkeys. Anat Rec A Discov Mol Cell Evol Biol 285:619-627.

Collins DL, Neelin P, Peters TM, Evans AC (1994) Automatic 3D intersubject registration of MR volumetric data in standardized Talairach space. J Comput Assist Tomogr 18:192-205.

Corbetta M, Shulman GL (2002) Control of goal-directed and stimulusdriven attention in the brain. Nat Rev Neurosci 3:201-215.

Corbetta M, Miezin FM, Shulman GL, Petersen SE (1993) A PET study of visuospatial attention. J Neurosci 13:1202-1226.

Cox LA Jr (1996) Reassessing benzene risks using internal doses and MonteCarlo uncertainty analysis. Environ Health Perspect 104:1413-1429.

Delorme A, Makeig S (2004) EEGLAB: an open source toolbox for analysis of single-trial EEG dynamics. J Neurosci Methods 134:9-21.

Desimone R, Duncan J (1995) Neural mechanisms of selective attention. Annu Rev Neurosci 18:193-222.

Dias EC, Bruce CJ (1994) Physiological correlate of fixation disengagement in the primate's frontal eye field. J Neurophysiol 72:2532-2537.

Dorris MC, Munoz DP (1995) A neural correlate for the gap effect on saccadic reaction times in monkey. J Neurophysiol 73:2558-2562.

Dorris MC, Munoz DP (1998) Saccadic probability influences motor preparation signals and time to saccadic initiation. J Neurosci 18:7015-7026.

Dorris MC, Paré M, Munoz DP (1997) Neuronal activity in monkey superior colliculus related to the initiation of saccadic eye movements. J Neurosci 17:8566-8579.

Driver J, Frith C (2000) Shifting baselines in attention research. Nat Rev Neurosci 1:147-148.

Dyckman KA, McDowell JE (2005) Behavioral plasticity of antisaccade performance following daily practice. Exp Brain Res 162:63-69.

Dyckman KA, Camchong J, Clements BA, McDowell JE (2007) An effect of context on saccade-related behavior and brain activity. Neuroimage 36: $774-784$.

Edelman JA, Keller EL (1996) Activity of visuomotor burst neurons in the superior colliculus accompanying express saccades. J Neurophysiol 76:908-926.

Ekstrom LB, Roelfsema PR, Arsenault JT, Bonmassar G, Vanduffel W (2008) Bottom up dependent gating of frontal signals in early visual cortex. Science 321:414-417.

Ergenoglu T, Demiralp T, Bayraktaroglu Z, Ergen M, Beydagi H, Uresin Y (2004) Alpha rhythm of the EEG modulates visual detection performance in humans. Cogn Brain Res 20:376-383.

Everling S, Munoz DP (2000) Neuronal correlates for preparatory set associated with prosaccades and antisaccades in the primate frontal eye field. J Neurosci 20:387-400.

Everling S, Krappmann P, Spantekow A, Flohr H (1996) Cortical potentials during the gap prior to express saccades and fast regular saccades. Exp Brain Res 111:139-143.

Ferree TC, Luu P, Russell GS, Tucker DM (2001) Scalp electrode impedance, infection risk, and EEG data quality. Clin Neurophysiol 112:536-544.

Fine I, Jacobs RA (2002) Comparing perceptual learning tasks: a review. $J$ Vis 2:190-203.

Fischer B, Boch R (1983) Saccadic eye movements after extremely short reaction times in the monkey. Brain Res 260:21-26.

Fischer B, Ramsperger E (1986) Human express saccades: effects of randomization and daily practice. Exp Brain Res 64:569-578.

Fischer B, Weber H, Biscaldi M, Aiple F, Otto P, Stuhr V (1993) Separate populations of visually guided saccades in humans: reaction times and amplitudes. Exp Brain Res 92:528-541.

Forman SD, Cohen JD, Fitzgerald M, Eddy WF, Mintun MA, Noll DC (1995) Improved assessment of significant activation in functional magnetic res- onance imaging (fMRI): use of a cluster-size threshold. Magn Reson Med 33:636-647.

Fuchs M, Wagner M, Kastner J (2001) Boundary element method volume conductor models for EEG source reconstruction. Clin Neurophysiol 112:1400-1407.

Hämäläinen MS, Ilmoniemi RJ (1994) Interpreting magnetic fields of the brain: minimum norm estimates. Med Biol Eng Comput 32:35-42.

Jin Y, O'Halloran JP, Plon L, Sandman CA, Potkin SG (2006) Alpha EEG predicts visual reaction time. Int J Neurosci 116:1035-1044.

Keating EG, Gooley SG, Pratt SE, Kelsey JE (1983) Progression in neuronal processing for saccadic eye movements from parietal cortex area lip to superior colliculus. Brain Res 269:143-149.

Kirschfeld K, Feiler R, Wolf-Oberhollenzer F (1996) Cortical oscillations and the origin of express saccades. Proc Biol Sci 263:459-468.

Krusemark EA, Keith Campbell W, Clementz BA (2008) Attributions, deception, and event related potentials: an investigation of the self-serving bias. Psychophysiology 45:511-515.

Leigh RJ, Zee DS (2006) The neurology of eye movements, Vol 4. Oxford: Oxford UP.

Loftus GR, Masson MEJ (1994) Using confidence intervals in withinsubject designs. Psychon Bull Rev 1:476-490.

Luna B, Thulborn KR, Strojwas MH, McCurtain BJ, Berman RA, Genovese CR, Sweeney JA (1998) Dorsal cortical regions subserving visually guided saccades in humans: an fMRI study. Cereb Cortex 8:40-47.

Lynch JC, Graybiel AM, Lobeck LJ (1985) The differential projection of two cytoarchitectonic subregions of the inferior parietal lobule of macaque upon the deep layers of the superior colliculus. J Comp Neurol 235:241-254.

Maclean CJ, Morton NE, Elston RC, Yee S (1976) Skewness in commingled distributions. Biometrics 32:695-699.

Mathewson KE, Gratton G, Fabiani M, Beck DM, Ro T (2009) To see or not to see: prestimulus alpha phase predicts visual awareness. J Neurosci 29:2725-2732.

McDowell JE, Dyckman KA, Austin B, Clementz BA (2008) Neurophysiology and neuroanatomy of reflexive and volitional saccades: evidence from studies of humans. Brain Cogn 68:255-270.

Mulaik SA, James LR, Van Alstine J, Bennett N, Lind S, Stilwell CD (1989) Evaluation of goodness-of-fit indices for structural equation models. Psychol Bull 105:430-445.

Munoz DP, Fecteau JH (2002) Vying for dominance: dynamic interactions control visual fixation and saccadic initiation in the superior colliculus. Prog Brain Res 140:3-19.

Paré M, Munoz DP (1996) Saccadic reaction time in the monkey: advanced preparation of oculomotor programs is primarily responsible for express saccade occurance. J Neurophysiol 76:3666-3681.

Paré M, Wurtz RH (2001) Progression in neuronal processing for saccadic eye movements from parietal cortex area lip to superior colliculus. J Neurophysiol 85:2545-2562.

Pascual-Marqui RD (2002) Standardized low-resolution brain electromagnetic tomography (sLORETA): technical details. Methods Find Exp Clin Pharmacol 24:5-12.

Pessoa L, Kastner S, Ungerleider LG (2003) Neuroimaging studies of attention: from modulation of sensory processing to top-down control. J Neurosci 23:3990-3998.

Ruff CC, Blankenburg F, Bjoertomt O, Bestmann S, Freeman E, Haynes JD, Rees G, Josephs O, Deichmann R, Driver J (2006) Concurrent TMSfMRI and psychophysics reveal frontal influences on human retinotopic visual cortex. Curr Biol 16:1479-1488.

Saslow MG (1967) Effects of components of displacement-setup stimuli upon latency for saccadic eye movements. J Opt Soc Am 57:1024-1029.

Schroeder CE, Lakatos P (2009) Low-frequency neuronal oscillations as instruments of sensory selection. Trends Neurosci 32:9-18.

Skrandies W, Anagnostou E (1999) Electroencephalographic cortical oscillations and saccadic eye movements in humans. Neurosci Lett 26:57-60.

Soufflet L, Boeijinga PH (2005) Linear inverse solutions: simulations from a realistic head model in MEG. Brain Topogr 18:87-99.

Tenke CE, Kayser J (2001) A convenient method for detecting electrolyte bridges in multichannel electroencephalogram and event-related potential recordings. Clin Neurophysiol 112:545-550. 